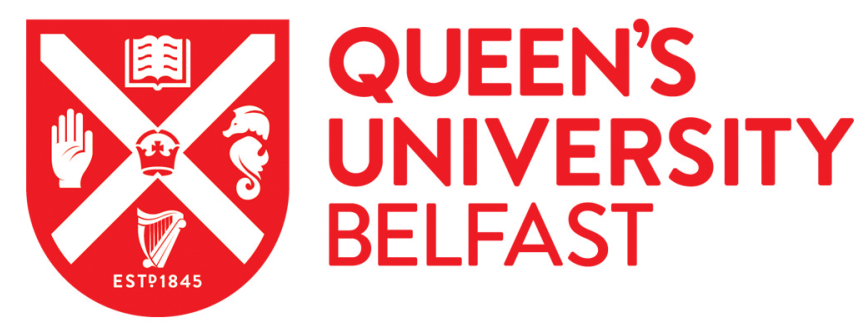

\title{
Awareness, Understanding and Treatment Practices when Managing Cachexia in End-Stage Kidney Disease
}

McKeaveney, C., Noble, H., de Barbieri, I., Strini, V., Maxwell, A. P., \& Reid, J. (2020). Awareness,

Understanding and Treatment Practices when Managing Cachexia in End-Stage Kidney Disease. Journal of Renal Care, 46(1), 35-44. https://doi.org/10.1111/jorc.12301

Published in:

Journal of Renal Care

Document Version:

Peer reviewed version

Queen's University Belfast - Research Portal:

Link to publication record in Queen's University Belfast Research Portal

Publisher rights

Copyright 2019 Wiley. This work is made available online in accordance with the publisher's policies. Please refer to any applicable terms of use of the publisher.

\section{General rights}

Copyright for the publications made accessible via the Queen's University Belfast Research Portal is retained by the author(s) and / or other copyright owners and it is a condition of accessing these publications that users recognise and abide by the legal requirements associated with these rights.

Take down policy

The Research Portal is Queen's institutional repository that provides access to Queen's research output. Every effort has been made to ensure that content in the Research Portal does not infringe any person's rights, or applicable UK laws. If you discover content in the Research Portal that you believe breaches copyright or violates any law, please contact openaccess@qub.ac.uk. 


\section{AWARENESS, UNDERSTANDING AND TREATMENT PRACTICES WHEN MANAGING CACHEXIA IN END-STAGE KIDNEY DISEASE}

Background: Cachexia is a wasting syndrome found within a range of chronic illnesses/life-limiting conditions, however awareness and understanding of cachexia amongst renal Health Care Professionals has not been investigated.

Objectives: To ascertain the awareness, understanding and treatment practices of Health Care Professionals who provide care for people with cachexia and endstage renal disease.

Methods: Health Care Professionals were recruited via the European Dialysis and Transplant Nurses Association/European Renal Care Association in September 2018. This was an exploratory study using a mixed-methods approach with those who provide care for patients with end-stage renal disease and cachexia. An online survey and two focus groups were conducted. Descriptive statistics and inductive thematic analysis were used to explore current knowledge and practices in renal cachexia.

Results: A total of 93 participants from 30 countries completed the online survey. Twelve Health Care Professionals agreed to participate in the focus groups. Reduced appetite, weight loss and muscle loss in relation to cachexia were accurately described, but the percentage of weight loss was unknown. The importance of multi-professional collaboration was recognised; however, the current management of cachexia was wide-ranging. Quality of life, patient-clinician communication and specialist support for carers were regarded as vital.

Conclusion: Timely identification and management of cachexia are needed to improve the quality of life for patients and appropriately support families. In order for these goals to be achieved, there is a need to increase awareness and understanding of cachexia amongst renal nurses.

\section{Keywords:}

cachexia

clinical practice

diagnosis

end-stage renal disease

health care professionals

international survey

loss of appetite

nursing

weight loss 


\section{INTRODUCTION}

Cachexia is recognised as a syndrome that is associated with chronic diseaserelated malnutrition with inflammation, highlighting specific nuances of the condition compared with other malnutrition disorders (Cederholm et al. 2017). Cachexia is a syndrome found within a range of chronic illnesses/life-limiting conditions, including cancer, cardiac and renal disease, and is known to affect approximately nine million patients globally (Farkas et al. 2013; Arthur et al. 2014). The generic definition of cachexia in chronic illness by Evans et al. (2008) identified weight loss or a body mass index (BMI) $<20 \mathrm{~kg} / \mathrm{m}^{2}$ and at least three out of five clinical or laboratory criteria: decreased muscle strength, fatigue, anorexia, low fatfree mass, inflammation, anaemia or low serum albumin. It is associated with increased morbidity, premature mortality, and significantly impacts on quality of life (QoL) due to associated anorexia, fatigue and decreased physical function (Muscaritoli et al. 2016). Early identification and accurate diagnosis are vital to improve health outcomes and overall survival rates (Argilés et al. 2014) however, recent research highlights a continuing lack of awareness and understanding of cachexia by health care professionals (HCPs) across many disciplines (O'Donoghue et al. 2018). Cachexia research has largely focused on patients with cancer or other end-of-life populations (Fearon et al. 2015; Ter Beek et al. 2016), however, no studies have investigated the awareness and understanding of cachexia amongst renal HCPs. It is important to identify such gaps in knowledge to help inform and improve current clinical practice within renal health care.

\section{LITERATURE REVIEW}

There is a wide spectrum of nutritional disorders associated with kidney disease (Cederholm et al. 2017) whereby HCPs have an important role to play when caring for patients with or at risk of cachexia including nutritional screening, symptom management and psychological support to promoting behaviour change (Scott et al. 2016). Substantial work has led to the development of a universally accepted definition for cachexia, which aims to help clinicians to identify and treat the underlying metabolic and nutritional issues (Arends et al. 2017), however, current clinical guidelines have not been updated to reflect the redefinitions (ESPEN guidelines, Cano et al. 2009; NKF KDOQI guidelines, Abecassis et al. 2008). There is no uniform disease-specific consensus on optimal therapy for cachexia in endstage renal disease (ESRD) (Penet \& Bhujwalla 2015) and a continuing overlap of wasting disorders (Cederholm et al. 2019) has resulted in HCPs lacking knowledge and clarity on appropriate nutritional care and effective nutritional care interventions (Scott et al. 2016). It is essential that HCPs have standard concepts and terminology to be able to appropriately screen, assess and treat patients. Surveys using traditional measures of nutritional status indicate that muscle wasting is common among persons with ESRD, with up to $75 \%$ of adults undergoing maintenance dialysis affected (Mak et al. 2011). ESRD is an inflammatory state, associated with an increase in biomarkers of inflammation, e.g. $\mathrm{C}$ reactive protein, and can result in cachexia, with loss of muscle and fat stores. Weight loss is the prominent clinical feature defining cachexia ( $>5 \%$ or more weight loss over 6 months or $>10 \%$ or more over 12 months; Evans et al. 2008; Mak et 
al. 2011), however, limited data are available on the classification of cachexia in persons with ESRD who often exhibit symptoms of anorexia, loss of lean muscle mass and altered energy expenditure. These symptoms are consistent with the syndrome of cachexia observed in other chronic diseases including cancer, heart failure and acquired immune deficiency syndrome (Kalantar-Zadeh et al. 2003). While progress has been made in exploring the awareness, understanding and treatment practices of HCPs who manage cachexia in these conditions, there continues to be a lack of knowledge in ESRD. To date, no study has explored these aspects for HCPs managing cachexia in ESRD. The importance of this work is underscored by the negative impact that symptoms of cachexia have on the quality of life (QoL) and the association of cachexia with a substantially increased risk of premature mortality (Kalantar-Zadeh et al. 2013). Skeletal muscle mass loss associated with cachexia cannot be fully reversed by conventional nutritional support alone, emphasising the need for early and specialist interventions. Cachexia is a multifactorial syndrome associated with anorexia, weight loss, nutritional deficits and metabolic derangements that would require a multimodal therapy approach to effectively combat the negative impact of this disorder.

\section{THE STUDY}

\section{AIM}

The aim of this study was to explore the awareness, understanding, and treatment practices of HCPs who manage cachexia in persons with ESRD.

\section{DESIGN}

Descriptive statistics from the survey provided are summarised narratively. Focus groups were used (one in Italian and one in English) and analysed using an inductive thematic analysis.

\section{PARTICIPANTS}

A purposive sampling strategy was applied using members of the European Dialysis and Transplant Nurses Association/European Renal Care Association (EDTNA/ERCA). The EDTNA/ERCA is an international multi-disciplinary organisation established to address the special educational needs of HCPS working with renal patients. Members include nurses, physicians, social workers, dieticians, technicians, transplant co-ordinators and unit managers. An initial invitation to the survey was sent and opened by 818 of 2,197 EDTNA/ERCA Members in September 2017, with a follow-up reminder email sent in July 2018. Approximately $11 \%(n=93)$ of members that opened the email participated in the survey and subsequent participants of the survey agreed to take part in the focus groups $(n=12)$. The $11 \%$ response rate is considered typical for web surveys and can be treated as reliable when response quality is checked (Van Mol 2017).

\section{DATA COLLECTION}

\section{SURVEY}


An invitation email that contained a link to the online 15-minute survey was contained within an email sent by EDTNA/ERCA to its members. The survey consisted of 13 multiple-choice questions and covered several relevant areas including weight loss, management of cachexia, and key goals of treatment. Ninety-three participants from 30 different countries completed the online survey. The basis of the survey was taken from Muscaritoli et al. (2016), with permission from the authors and adapted for the renal population. The questionnaire has not been externally validated as it has been administrated only by the original authors and has not been used in a renal population.

\section{FOCUS GROUP}

At the end of the survey, participants were asked to forward contact information if consenting to take part in a future focus group. Twelve HCPs (seven Italian and five English participants) provided contact email addresses. Two 45-minute focus groups were conducted (one in Italian and one in English). Two experienced nephrology academics acted as moderator and note taker for the focus groups.

\section{ETHICAL CONSIDERATIONS}

Ethical approval was obtained from the University relevant to the study (04.18.M5.V2). Completion of the anonymous online survey was taken as consent. Those who completed the online survey were asked to click on a link that took them outside the anonymous survey to provide their contact details if they wished to be part of a focus group. The English-speaking focus group took place at the 47th EDTNA/ERCA International conference in Genoa, Italy and the Italian speaking focus group took place within the nephrology department at Padua Hospital, Italy, in September 2018. Informed consent was gained from all participants prior to the focus groups commencing.

\section{DATA ANALYSIS}

Quantitative survey data from the completed online surveys were exported into SPSS. Data from the survey were analysed using SPSS software (IBM Corp. Released 2016; IBM SPSS, Statistics for Windows, Version 24.0, Armonk, NY: IBM Corp). Descriptive statistics including frequencies and percentages were generated for each response. Analysis of the qualitative interviews was carried out using the framework of qualitative analysis developed by Huberman and Miles (2002) using an inductive approach to coding and analysis. This approach aims to clarify the data via data reduction and created meaning in the data through the development of summary themes or categories from raw data. Both focus groups were digitally recorded and transcribed verbatim, the Italian speaking focus group manuscript was translated into English. Data analysis was led by one researcher (CM) and themes identified were independently confirmed by a further author (JR).

\section{RIGOUR}

Participants from the two countries took part in the focus groups, unlike the survey which included participants from 30 countries. Therefore, maintaining the 
trustworthiness of the qualitative data collected involved method triangulation. To check and establish the validity of the findings, a comparison was made between the survey and focus group responses. Using multiple perspectives allowed verification of data (Onwuegbuzie \& Leech 2007). To ensure the transferability of qualitative enquiry, the purposive sampling strategy used in this study ensured key informants were included to provide important knowledge of the issues under investigation (Anney 2014).

\section{FINDINGS}

\section{SURVEY}

Table 1. Demographic and professional characteristics of participants

\begin{tabular}{l|ll}
\multicolumn{2}{l}{ Characteristics } & \multicolumn{1}{l}{$\boldsymbol{n = 9 3}(\%)$} \\
\hline Gender & Female & $78(72 \%)$ \\
& $25-34$ & 14 \\
Age, mean (SD), years & $35-44$ & 33 \\
& $45-54$ & 28 \\
& $55-64$ & 15 \\
Profession & $65-74$ & 3 \\
& Nursing & $84(90.3 \%)$ \\
& Doctor & $2(2.2 \%)$ \\
Time in current role, years & Dietician & $5(5.3 \%)$ \\
& Other & $2(2.2 \%)$ \\
& $<5$ & $5(5.4 \%)$ \\
& $5-10$ & $9(9.7 \%)$ \\
& $10-15$ & $10(10.8 \%)$ \\
& $15-20$ & $27(29.0 \%)$ \\
& $20-25$ & $11(11.8 \%)$ \\
& $>25$ & $31(33.3 \%)$
\end{tabular}

Table 1 reports the demographic and professional characteristics for participants who completed the online survey. The respondents were predominately female $(72 \% ; n=78)$ and those from the nursing profession $(90 \% ; n=84)$. The majority of participants were between 35 and 44 years of age. Only 5\% of participants had worked in their current role for less than 5 years $(n=5), 61 \%(n=57)$ of participants between 5 and 25 years. A large number of participants had worked in their current role for more than 25 years $(33 \% ; n=31)$. 
Figure 1. Country of origin of participants

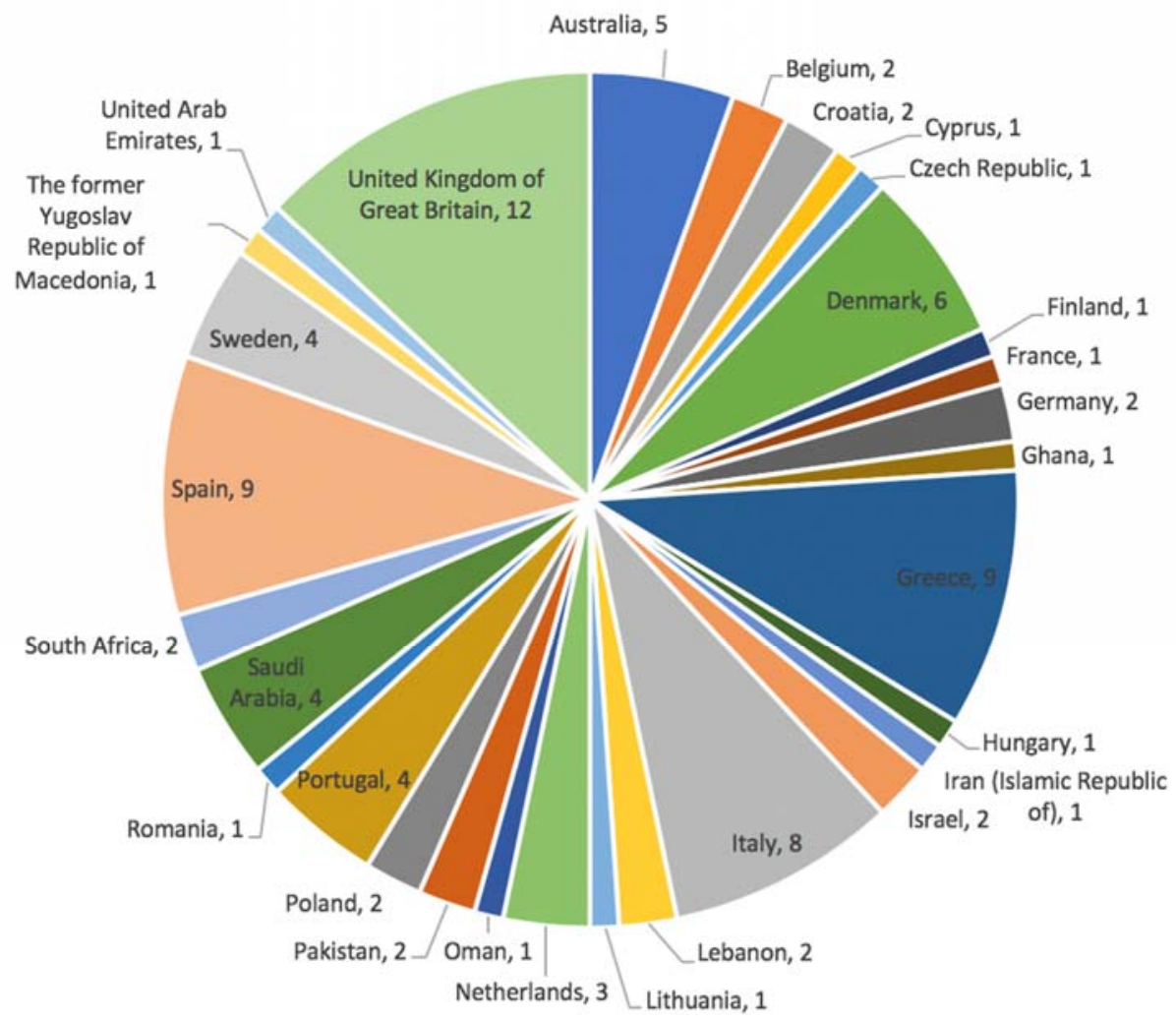

Thirty countries participated in the survey, as can be seen in Figure 1 . The majority of respondents were from the UK $(n=12 ; 13 \%)$ followed by Spain $(n=9 ; 10 \%)$, Greece $(n=9 ; 10 \%)$, Italy $(n=8 ; 9 \%)$, Denmark $(n=6 ; 7 \%)$ and Australia $(n=5$; $5 \%$ ). Four HCPs participated from the following countries: Sweden, Portugal and Saudi Arabia. Three HCPs participated from the Netherlands. Two HCPs participated from the following countries: South Africa, Poland, Pakistan, Belgium, Croatia, Germany, Lebanon and Israel. One HCP participated from the following countries: Romania, The Former Yugoslav Republic of Macedonia, United Arab Emirates, Cyprus, Czech Republic, France, Finland, Ghana, Hungary, Lithuania, Oman and Iran.

Figure 2. Survey question: What indicators do you consider to be part of cachexia in renal disease? Select all that apply. 


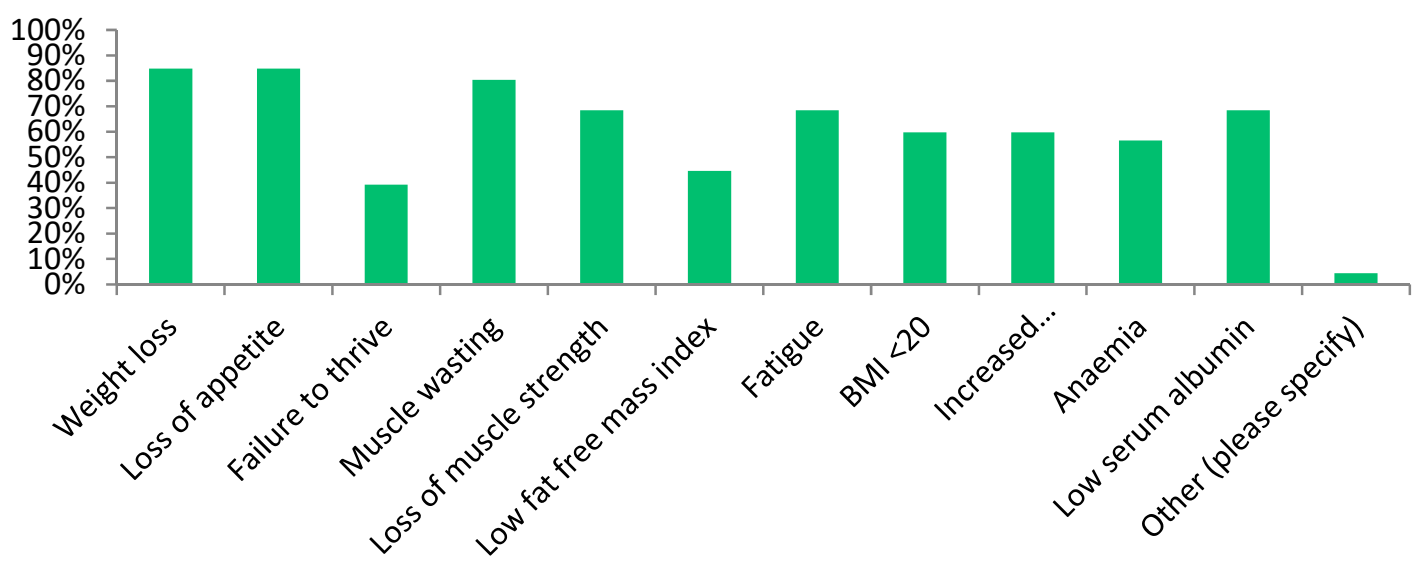

The most commonly reported symptoms of cachexia by HCPs (Figure 2) were weight loss and anorexia (loss of appetite, 85\%). This was followed by key indicators; muscle wasting (80\%), loss of muscle strength, fatigue and low serum albumin (68\%), BMI $<20 \mathrm{~kg} / \mathrm{m} 2$ and increased inflammatory markers (60\%), anaemia (57\%) and failure to thrive (39\%). Additional answers included "depression" and "satiate molecules as leptin not being removed by standard HD that might be removed more effectively with HDF" and "decreased physical activity".

Figure 3. Survey question: in which cases have you seen a treatment for cachexia being prescribed? Select all that apply.

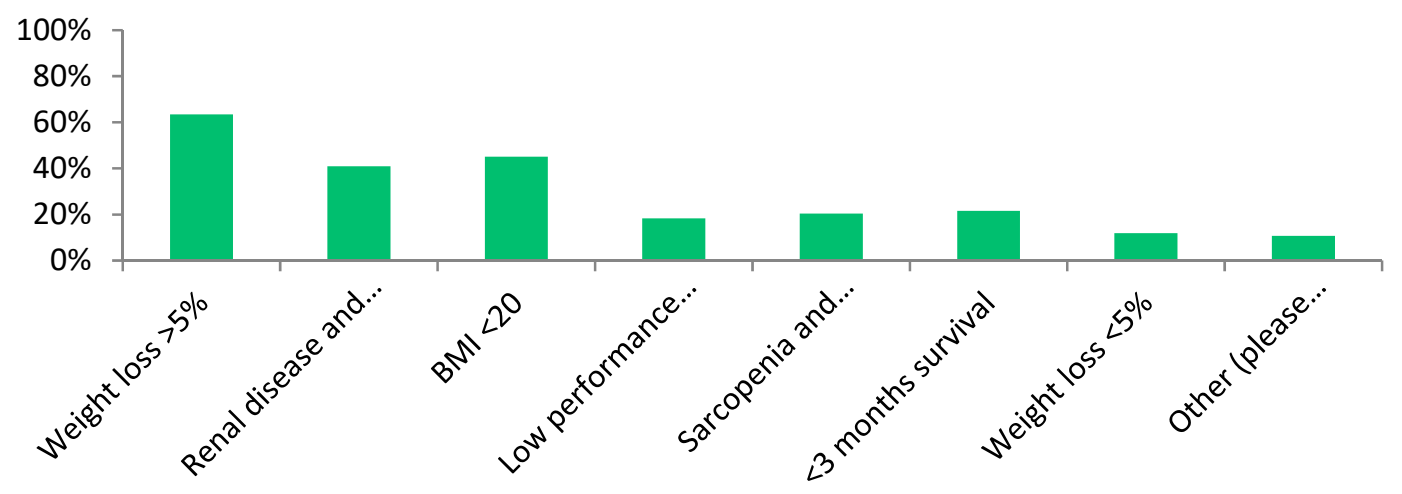

The primary factor leading to a treatment for cachexia being prescribed (Figure 3) was weight loss > 5\% (63\%) followed by BMI < $20 \mathrm{~kg} / \mathrm{m} 2(45 \%)$ and "renal disease and both procatabolic + non-responsive to treatment" (41\%). This was followed by $<3$ month survival (22\%), sarcopenia and weight loss $>2 \%$ (20\%), low performance score (18\%), weight loss $<5 \%(12 \%)$. Additional answers included "muscle wasting, loss of appetite, low albumin", "low albumin", "have not seen treatment for cachexia being prescribed", "chronic inflammation", "declining SGA score", "hard to eat" and "albumin < 4". 
Figure 4. Survey question: At what percentage of (involuntary) weight loss from baseline do you consider a patient to be cachectic and need to start treatment?

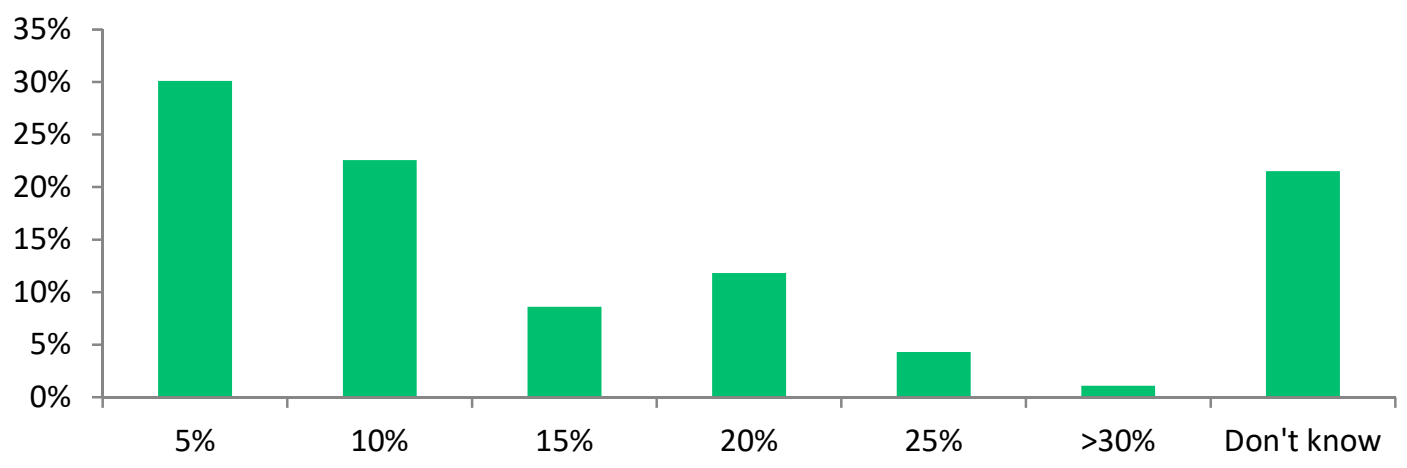

The majority of participants reported that if patients had $>5 \%(30 \%)$ of involuntary weight loss from baseline, they would consider them to be cachectic and start treatment (Figure 4). This was followed by $>10 \%$ of involuntary weight loss (23\%), however, over one-fifth (22\%) of participants did not know what the percentage of involuntary weight loss from baseline is considered to represent cachexia requiring treatment. The remaining participants $(26 \% ; n=24)$ reported $>15 \%$ of involuntary weight loss or greater.

Figure 5. At what percentage of (involuntary) weight loss over the previous 12 months would you consider a patient to be cachectic?

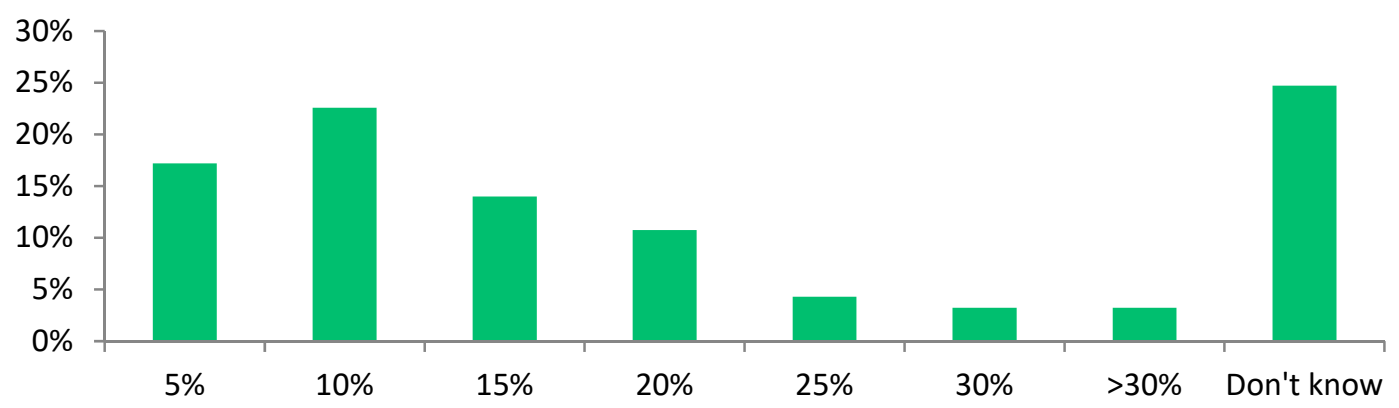

A quarter of participants $(n=23)$ did not know what percentage of (involuntary) weight loss over the previous 12 months would indicate a cachexic patient. This was followed by $23 \%$ of participants who correctly reported $10 \%(n=21)$. Others reported $5 \%$ weight loss $(17 \% ; n=16)$ and $15 \%(14 \% ; n=13)$. The remaining participants $(n=20)$ reported involuntary weight loss of $20 \%$ or greater over the previous 12 months. 
Figure 6. What are the factors that are considered in clinical practice when an agent is being used to treat cachexia in renal disease and rate the importance of each factor?

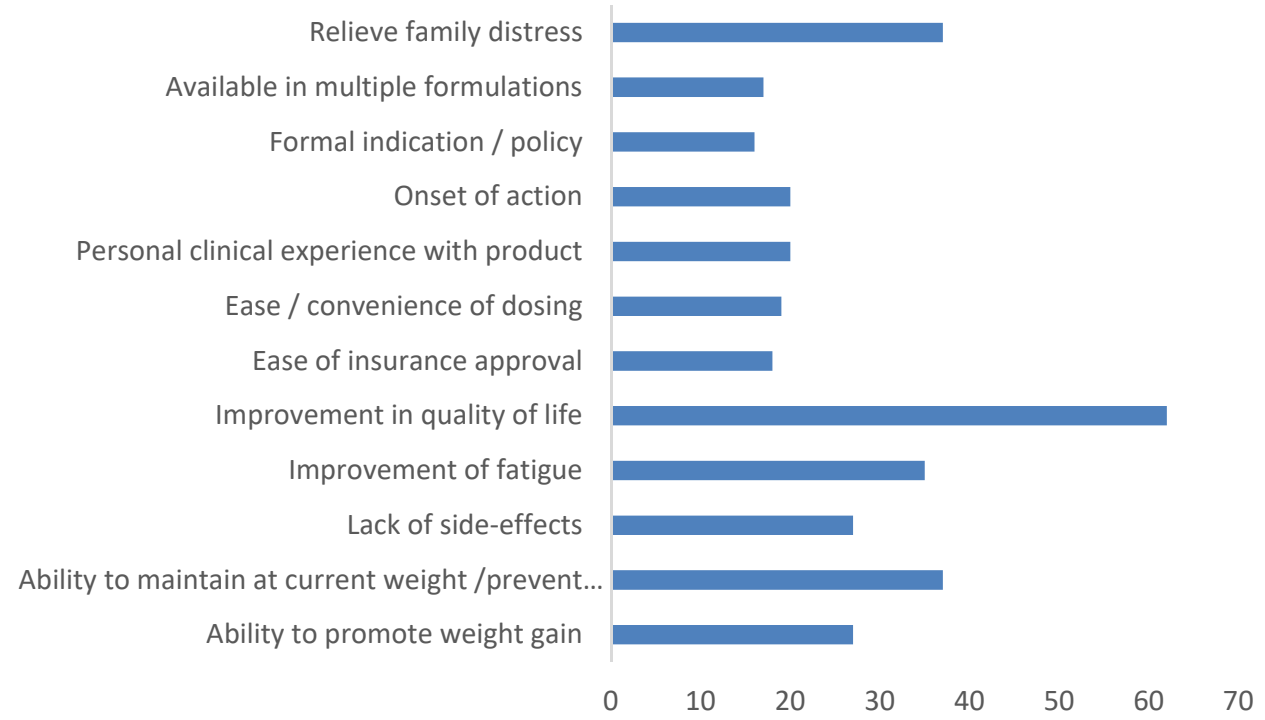

An improvement in the quality of life was rated as the most important factor (69\%) when an agent is being used to treat cachexia in ESRD (Figure 6). This was followed by relieving family distress (41\%) in addition to the ability to maintain the patient at current weight/prevent further weight loss (40\%), improve fatigue (39\%).

Figure 7. In your opinion what are they key goals of first line treatment for cachexia in renal disease? Select all that apply.

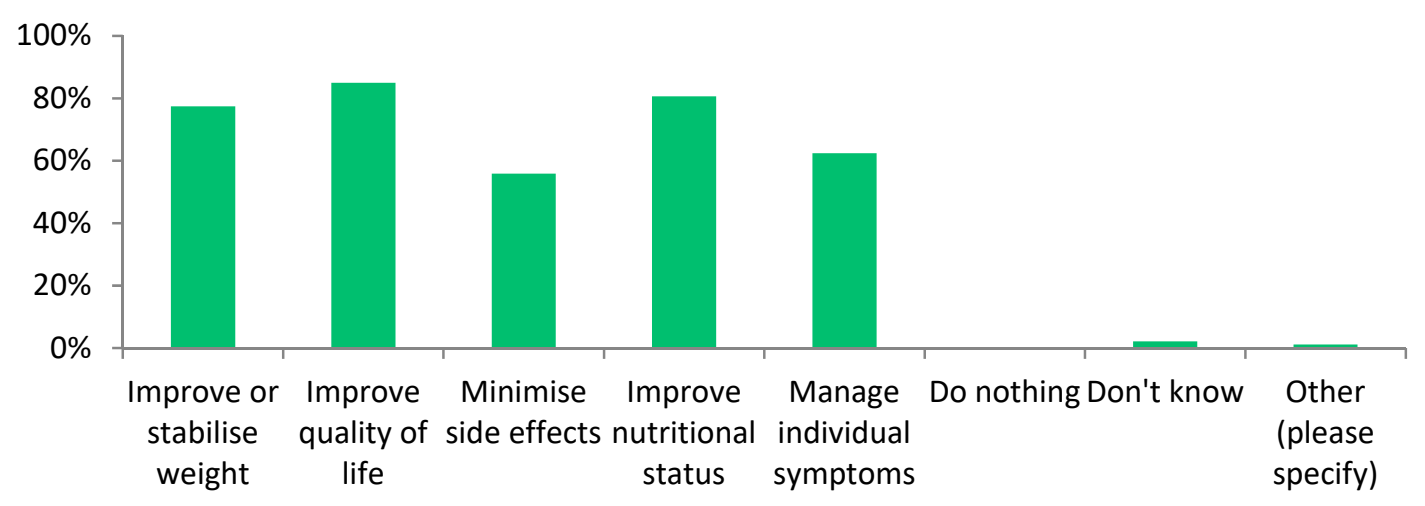

As can be seen in Figure 7, the primary goal of first-line treatment for cachexia in kidney disease was improving quality of life (85\%) followed by improvement in nutritional status (81\%) and improve or stabilise weight (77\%). Managing individual symptoms (62\%) and minimising side effects (56\%) were rated next. Only two 
participants responded that they "don't know" what to do and no participants reported they would do nothing. An additional (other) answer included "patients must have good dialysis adequacy".

Figure 8. What improvements would you like to see in the treatment of renal cachexia? Select all that apply.

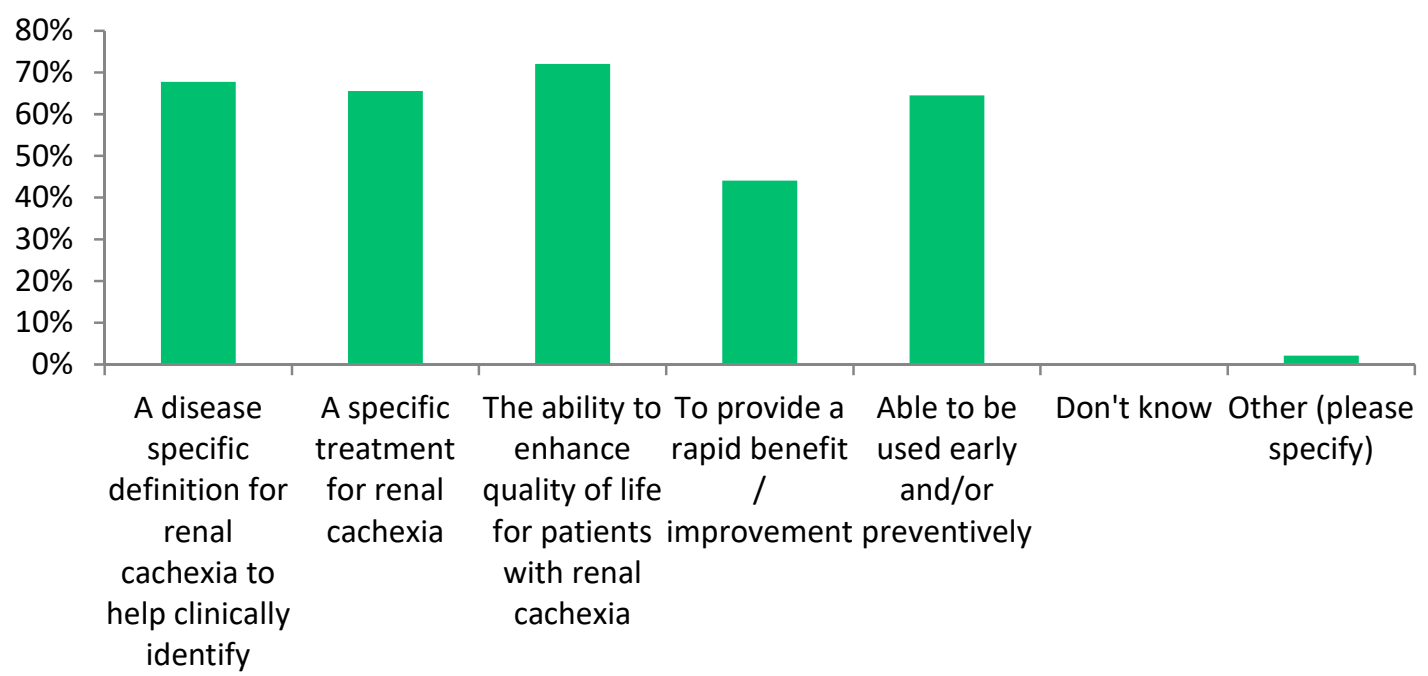

When asked what improvements they would like to see in the treatment of renal cachexia (Figure 8), the majority of participants reported they would like the ability to improve quality of life for patients with renal cachexia (72\%) followed by a disease-specific definition for renal cachexia to help identify such patients (68\%). A specific treatment for renal cachexia was also reported (66\%), followed by a treatment that could be used early and/or preventively (65\%) and the ability to provide a rapid benefit or improvement of renal cachexia (44\%). Additional responses included "follow up with psychologist" and "to try HDF on this group of patients".

\section{FOCUS GROUP}

Key themes included: understanding cachexia; management of cachexia; and the impact on QoL for patients and carers. Each will be explored in turn with excerpts provided.

\section{UNDERSTANDING CACHEXIA}

The term "cachexia" was associated with negative outcomes. Imagery of "frail" patients who had, muscle mass reduction, a "flaccid appearance" and were approaching end-of-life was conveyed by participants; 
"...somebody heading towards end of life, they have started to lose a lot of weight, I sort of visualise in the bed that little person...dialysis is nearly done, they are really struggling to maintain their bodyweight and...with life I guess."

Lack of oral intake and weight loss, despite issues with fluid retention that are specific to this chronic illness, were often portrayed as characteristics of cachexia;

"...not eating well, often despite that fact, they have gained all that fluid weight...so all of a sudden they are cachectic...then sort of just end of life body failures."

The preferential loss of lean muscle mass associated with cachexia and its impact on physical ability were also highlighted, as was the multi-dimensional impact of cachexia;

"Somebody that hasn't got any energy, often depressed and just very flat, cannot fulfil his physical activity or duty, generally weak, sometimes anxious, can't cope. Risk of infection or having infection."

\section{MANAGEMENT OF CACHEXIA}

The current management of patients with ESRD and cachexia was described as poor;

"You know everybody knows its [renal cachexia] management inadequate..."

HCPs agreed they had an important role to play in the prevention, diagnosis and treatment of cachexia where there are routine opportunities to intervene in relation to maintenance haemodialysis patients;

“...I think we forget that sometimes with these [day] patients that are not admitted to our ward, we see them 3 times a week in our haemodialysis unit, maybe now we should start looking at the restriction."

HCPs acknowledged that a lack of guidelines or standards of care for the treatment of renal cachexia made current practice wide-ranging and superficial;

"There are no evaluation scales [for renal cachexia], they are sometimes evaluated by dieticians. Nurses talk about evaluation scales, but they not do not use them."

Cachexia was depicted as a complex condition that required specialised care, but the signs of cachexia were often overshadowed by other conditions causing a delay in intervention;

"...the real reason [nurses] pick up [on cachexia] is ultimately often the patient has never been noted to be losing weight...suddenly they go into pulmonary oedema and somebody is saying oh my goodness they have got 8 kilos of fluid and it turns out they have actually lost $6 \mathrm{~kg}$ of bodyweight... 
that is not an unusual occurrence in a dialysis unit unfortunately."

Participants emphasised the need for a multi-professional approach when identifying and treating patients with cachexia. However, factors such as funding were highlighted as having a substantial impact on appropriate patient care. Without a specialist team there was risk of failing to prevent, diagnosis, provide timely treatment and improve quality;

"Nutritional screening and [a] physician and also involve a social worker...the social worker can improve their psychological status...[help patients] follow the orders and advice of the nutritionist..."

An integrated multi-professional approach model, seen within cancer care, was considered as ideal for ESRD patients;

“...there are all sorts of support that cancer patients get which other patients don't automatically get...there is a team. I can see it working so well for cancer and it is almost looking like you could create a similar model for renal patients."

\section{THE IMPACT ON QOL FOR PATIENTS AND CARERS}

HCPs acknowledged the impact of cachexia on QoL as extensive, and that it continues to be an unmet need in the care of patients with kidney disease;

"Counselling in all honesty, usually if someone is cachectic there is usually something underneath happening, ... a lot of them are either depressed or maybe they are getting to the stage that they should come off dialysis so really talking to them and understanding what's going on and why they are losing weight and the reasons..."

Food and eating are also fundamental aspects of QoL that are impacted by ESRD. It was also recognised by HCPs that "information material is scarce or absent" and this lack of information can mean that patients are "very frightened" about changes in appetite that may be associated with cachexia:

"...do they just not want food anymore......so I think really talking to the patients is the first step to trying to understand what's going on and do they want to change and what can we put in place to change it".

When family involvement was absent, it was seen as a disadvantage for the patient and the HCPs involved;

"Very often a lot of families don't come in...patients [are] brought in by community transport... if you challenge them on what they are eating they will just tell you what they think you are wanting to hear." 
Participants explained that the management of food can cause significant distress for families and carers of patients with cachexia. In many cases, confusion around renal diet restrictions coupled with cachexia was seen;

"(Relatives and patients) get hung up and confused about the restrictions... paranoid about their potassium. It is drilled into them from nowhere that they have got to watch their potassium so consequently they won't eat any foods with potassium...gets really complicated in their heads, they just get confused and a lot of their families too."

Indeed, lack of eating was a barometer of health and carers could interpret cachexia as a sign that the end-of-life was approaching, causing considerable distress;

"My experience is that [relatives] get very anxious... because they think, now we are at the end of life...suffering cachexia you know, now it is very bad."

\section{DISCUSSION}

A lack of uniform clinical knowledge and an absence of diagnostic criteria for cachexia in patients with ESRD hinders both research and clinical management of this condition and is likely to have a negative impact on a range of health outcomes including patient survival (Laviano et al. 2015). Therefore, it is important to assess awareness and understanding of cachexia to help improve early identification, treatment and support for patients and their families.

The survey provides insights into the views of HCPs, the majority of whom are from a nursing background, on the range of prognostic indicators of cachexia associated with ESRD. The observation of weight loss as a prognostic indicator for cachexia, alongside loss of appetite and muscle wasting, demonstrates many HCPs are able to discriminate between renal cachexia and other forms of weight loss such as starvation (Cuppari et al. 2014). However, specific nuances of cachexia, such as the percentage of weight loss indicative of a diagnosis of cachexia was not known and can have consequences for when management strategies can be started.

Conversely, a reliance on weight loss alone could result in delayed intervention and irreversible health outcomes. Discrimination of cachectic prognostic factors can be difficult in ESRD due to associated co-morbidities, or the dialysis process itself. This emphasises the importance of understanding the nutritional and metabolic complexities in renal cachexia to reach the correct diagnosis.

This study, similar to other disease groups (Fearon et al. 2015), reported the management of cachexia as complex and challenging for HCPs compounded by a lack of guidelines or standards of practice for the treatment of renal cachexia (Reid et al. 2010). Good quality cachexia management involves multi-professional resources. This includes dedicated dieticians and psychological support. In addition, timely standardised assessment, inclusion of families as well as 
evidence-based practice recommendations to reflect the multi-dimensional impact of cachexia are required (Reid et al. 2018). Models of care for cancer patients with cachexia were regarded as good examples that could be adapted for the management of patients with ESRD. Multidisciplinary rehabilitation models of care for cancer cachexia have been shown to improve QoL as well as improving survival rates (Ellis 2012). Additionally, specialist cachexia clinics, for cancer patient populations, provide the assessment and expertise needed by individuals with the cachexia syndrome (Del Fabbro et al. 2011).

The role of QoL was strongly acknowledged as a key factor in the identification, treatment and support of cachexia. Research has shown QoL is significantly impaired in patients with ESRD (Joshi 2014). Depression, sleep disorders and sexual dysfunction as well as a range of associated co-morbidities (e.g. diabetes; Pagels et al. 2012) impacting on QoL are commonly reported in ESRD (Stenvinkel et al. 2015). ESRD treatment is also associated with reduced QoL in caregivers (Goff et al. 2015). The management of ESRD involves significant lifestyle changes for both patients and caregivers. Problematic areas identified in this study include encouraging food intake. Such issues are reported as central to caregiving and can cause conflict and distress (Reid et al. 2009). Families also have an important role to play, as found in this study, as they help patients engage with clinical teams and their absence can have a negative impact. Research demonstrates that family support can improve treatment transition and increase compliance rates (Dobrev et al. 2001). Thus, underpinning the importance of supporting families in parallel with patients.

This is the first qualitative study eliciting the views of HCPs about cachexia in renal disease. Whereas anecdotal evidence suggested that diagnosis and treatment was ad-hoc, this study provides an evidence base in relation to this and in particular how the lack of evidence-based guidelines hinders timely management. Additionally, this study highlights that similar to cachexia in other chronic illnesses, it negatively impacts on both patients and their lay carers.

\section{LIMITATIONS}

This study has limitations relating to the smaller than anticipated survey sample size. Although over 2000 registered members of the EDTNA/ERCA were invited to take part, we cannot be sure all access their emails regularly, and if the invitation reached all members. That said, of the 818 that did open their email, an 11\% response rate is typical for web surveys and can be treated as reliable when response quality is checked (Van Mol 2017). Also, although 30 countries provided responds to our survey, only two countries participated in the focus group and the majority of respondents were from one professional discipline (nursing). This study was also limited to EDTNA/ERCA members which reduced generalisability of findings. These limitations highlight potential biases including the representativeness of participants and the lack of generalisation to other HCPs and countries. 


\section{CONCLUSION}

This is the first international study to examine the awareness and understanding of cachexia in ESRD within renal HCPs. There were 30 countries participating which provides a novel worldwide perspective. The HCPs clearly understood the implications of cachexia including physical, psychological and social factors and recognised the need to identify uniformed disease-specific management for cachexia in ESRD. Overall, this information helps to inform the learning and support needs of renal HCPs. Our findings have identified some knowledge gaps for HCPs when attempting to recognise cachexia in ESRD patients. This could be remedied by targeted education on cachexia and its impact in ESRD. It is essential that HCPs understand the multifactorial nature of cachexia and apply it consistently in clinical practice.

However, more research is needed to investigate models of cachexia management in this population, working towards a uniform criteria for screening, diagnosis and treatment to update relevant clinical guidelines (Kokura et al. 2017).

\section{ACKNOWLEDGEMENTS}

Thanks are given to the members of the European Dialysis and Transplant Nurses Association/European Renal Care Association (EDTNA/ERCA).

\section{CONFLICT OF INTERESTS}

The authors declare that there are no conflict of interests.

\section{AUTHOR CONTRIBUTIONS}

$\mathrm{CM}, \mathrm{HN}, \mathrm{IB}, \mathrm{VS}, \mathrm{JR}$ : Made substantial contributions to conception and design, or acquisition of data, or analysis and interpretation of data; CM, HN, IB, VS, JR, APM: Involved in drafting the manuscript or revising it critically for important intellectual content; CM, HN, IB, VS, JR, APM: Given final approval of the version to be published. Each author should have participated sufficiently in the work to take public responsibility for appropriate portions of the content; CM, HN, JR: Agreed to be accountable for all aspects of the work in ensuring that questions related to the accuracy or integrity of any part of the work are appropriately investigated and resolved. 


\section{References}

Abecassis M. et al. (2008). Kidney transplantation as primary therapy for endstage renal disease: a National Kidney Foundation/Kidney Disease Outcomes Quality Initiative (NKF/KDOQI ${ }^{\mathrm{TM}}$ ) conference. Clinical Journal of the American Society of Nephrology, 3(2), 471-480.

Arends J., Bachmann P., Baracos V. et al. (2017). ESPEN guidelines on nutrition in cancer patients. Clinical Nutrition, 36(1), 11-48.

Arthur S. T., Noone J. M., Van Doren B. A. et al. (2014). One-year prevalence, comorbidities and cost of cachexia-related inpatient admissions in the USA. Drugs in context, 3.

Argilés J. M., Busquets S., Stemmler B. et al. (2014). Cancer cachexia: understanding the molecular basis. Nature reviews Cancer, 14(11), 754.

Anney V. N. (2014) Ensuring the quality of the Journal of Emerging Trends in Educational Research and Policy Studies, 5(2), 272-281

Cano N.J.M. et al. (2009). ESPEN Guidelines on Parenteral Nutrition: adult renal failure. Clinical Nutrition, 28(4), 401-414.

Cederholm T., Barazzoni R., Austin P. et al. (2017). ESPEN guidelines on definitions and terminology of clinical nutrition. Clinical Nutrition, 36(1), 49-64.

Cederholm T., Jensen G.L., Correia M.I.T. et al. (2019). GLIM criteria for the diagnosis of malnutrition-A consensus report from the global clinical nutrition community. Journal of cachexia, sarcopenia and muscle, 10(1), 207-217.

Cuppari L., Meireles M. S., Ramos C. I. et al. (2014). Subjective global assessment for the diagnosis of protein-energy wasting in nondialysisdependent chronic kidney disease patients. Journal of Renal Nutrition, 24(6), 385-389.

Dobrev S. D., Kim T. Y. \& Hannan M. T. (2001). Dynamics of niche width and resource partitioning. American Journal of Sociology, 106(5), 1299-1337.

Del Fabbro E., Hui D., Dalal S. et al. (2011). Clinical outcomes and contributors to weight loss in a cancer cachexia clinic. Journal of palliative medicine, 14(9), 1004-1008.

Dobrev S. D., Kim T. Y. \& Hannan M. T. (2001). Dynamics of niche width and resource partitioning. American Journal of Sociology, 106(5), 1299-1337. 
Ellis P. M. (2012). The importance of multidisciplinary team management of patients with non-small-cell lung cancer. Current oncology, 19(Suppl 1), S7.

Evans W. J., Morley J. E., Argilés J. et al. (2008). Cachexia: a new definition. Clinical nutrition, 27(6), 793-799.

Farkas J., von Haehling S., Kalantar-Zadeh K. et al. (2013). Cachexia as a major public health problem: frequent, costly, and deadly. Journal of cachexia, sarcopenia and muscle, 4(3), 173-178.

Fearon K. C. H., Argiles J. M., Baracos V. E. et al. (2015). Request for regulatory guidance for cancer cachexia intervention trials. Journal of cachexia, sarcopenia and muscle, 6(4), 272-274.

Goff S. L., Eneanya N. D., Feinberg R. et al. (2015). Advance care planning: a qualitative study of dialysis patients and families. Clinical Journal of the American Society of Nephrology, CJN-07490714.

Huberman M. \& Miles M. B. (2002). The qualitative researcher's companion. Sage.

IBM Corp. Released 2016. IBM SPSS Statistics for Windows, Version 24.0. Armonk, NY: IBM Corp.

Joshi V. D. (2014). Quality of life in end stage renal disease patients. World journal of nephrology, 3(4), 308.

Kalantar-Zadeh, K., Ikizler T. A., Block G. et al. (2003). Malnutritioninflammation complex syndrome in dialysis patients: causes and consequences. American journal of kidney diseases, 42(5), 864-881.

Kalantar-Zadeh K., Rhee, C., Sim J. J. et al. (2013). Why cachexia kills: examining the causality of poor outcomes in wasting conditions. Journal of cachexia, sarcopenia and muscle, 4(2), 89-94.

Kokura Y., Wakabayashi H., Maeda, K. et al. (2017). Impact of a multidisciplinary rehabilitation nutrition team on evaluating sarcopenia, cachexia and practice of rehabilitation nutrition. The Journal of Medical Investigation, 64(1.2), 140-145.

Laviano A., Koverech A. \& Mari A. (2015). Cachexia: clinical features when inflammation drives malnutrition. Proceedings of the Nutrition Society, 74(4), 348-354.

Mak R. H., Ikizler A. T., Kovesdy C. P. et al. (2011). Wasting in chronic kidney disease. Journal of cachexia, sarcopenia and muscle, 2(1), 9-25. d 
Muscaritoli M., Rossi Fanelli F., \& Molfino A. (2016). Perspectives of health care professionals on cancer cachexia: results from three global surveys. Annals of Oncology, mdw420.

O'Donoghue N., Doyle, S., Walsh D. et al. (2018). Nutritional status in cancer: Perspectives of healthcare professionals. e18550-e18550.

Onwuegbuzie A. J. \& Leech N. L. (2007). Validity and qualitative research: An oxymoron? Quality \& Quantity, 41(2), 233-249.

Pagels A. A., Söderkvist B. K., Medin C. et al. (2012). Health-related quality of life in different stages of chronic kidney disease and at initiation of dialysis treatment. Health and quality of life outcomes, 10(1), 71.

Penet M. F. \& Bhujwalla Z. M. (2015). Cancer cachexia, recent advances, and future directions. Cancer journal (Sudbury, Mass.), 21(2), 117.

Reid J., McKenna H., Fitzsimons D. et al. (2009, July). Fighting over food: patient and family understanding of cancer cachexia. In Oncology nursing forum, 36 (4).

Reid J., Mc Kenna H. P., Fitzsimons D. et al. (2010). An exploration of the experience of cancer cachexia: what patients and their families want from healthcare professionals. European journal of cancer care, 19(5), 682-689.

Reid J., Noble H. R., Adamson G. et al. (2018). Establishing a clinical phenotype for cachexia in end stage kidney disease-study protocol. BMC nephrology, 19(1), 38.

Scott D., Reid J., Hudson P. et al. (2016). Health care professionals' experience, understanding and perception of need of advanced care patients with cachexia and their families: The benefits of a dedicated clinic. BMC Palliative Care 15, 100.

Stenvinkel P., Carrero J. J., Von Walden F. et al. (2015). Muscle wasting in end-stage renal disease promulgates premature death: established, emerging and potential novel treatment strategies. Nephrology Dialysis Transplantation, 31(7), 1070-1077.

Ter Beek L., Vanhauwaert E., Slinde F. et al. (2016). Unsatisfactory knowledge and use of terminology regarding malnutrition, starvation, cachexia and sarcopenia among dietitians. Clinical Nutrition, 35(6), 14501456. 
Van Mol C. (2017). Improving web survey efficiency: the impact of an extra reminder and reminder content on web survey response. International Journal of Social Research Methodology, 20(4), 317-327. 